\title{
Is the nonlinear Meissner effect unobservable?
}

\author{
M. -R. Li, ${ }^{a, b}$ P. J. Hirschfeld ${ }^{a, c}$ and P. Wölfle ${ }^{a}$ \\ ${ }^{a}$ Institut für Theorie der Kondensierten Materie, Universität Karlsruhe, 76128 Karlsruhe, Germany \\ ${ }^{b}$ Department of Physics, Nanjing University, Nanjing 210093, P.R.China \\ ${ }^{c}$ Department of Physics, University of Florida, Gainesville, FL 32611, USA
}

\begin{abstract}
We examine the effects of nonlocal electrodynamics for a $d$-wave superconductor on the field dependence of the magnetic penetration depth. The linear field dependence predicted in the local limit, commonly known as the nonlinear Meissner effect, is instead found to be quadratic, $\delta \lambda \sim H^{2}$ for fields below a crossover scale $H^{*}$. This crossover is shown to be geometry dependent, and for most orientations of the screening currents, is of the same order as or greater than $H_{c 1}$, implying that the nonlinear Meissner effect can not be observed. For special orientations where the current flows along the nodal directions, however, the nonlinear Meissner effect may be recovered.
\end{abstract}

PACS Numbers: 74.25.Nf, 74.20.Fg

Measurements of the linear term in the temperature dependence of the electromagnetic penetration depth [1] $\delta \lambda(T) \equiv \lambda(T)-\lambda(0) \sim T$ played a pivotal role in the identification of the $d$-wave symmetry of the cuprate superconductors. It was always clear, however, that they were sensitive only to the depletion of the superfluid fraction by the thermal excitations of quasiparticles with momenta near the order parameter nodes, and hence provided information only on the energy gap on these regions of the Fermi surface. Furthermore, in a $d$-wave superconductor with tetragonal crystal symmetry, the penetration depth is independent of the angle $\theta$ the supercurrent makes with the crystal axes since the linear response tensor is isotropic; the gap shape is thus not directly reflected in the angle dependence of the effect.

Yip and Sauls 2] argued, however, that information about the gap shape in an unconventional superconductor with line nodes could be obtained by measuring the nonlinear (magnetic field-dependent) penetration depth $\delta \lambda(H)=\lambda(H)-\lambda(0)$, and predicted an unusual field dependence,

$$
\frac{\delta \lambda(H)}{\lambda(0)} \simeq \frac{\zeta_{\theta}}{\sqrt{2}} \frac{H}{H_{0}}
$$

where $\zeta_{\theta}$ is a (weak) angle-dependent function, $H$ is the applied magnetic field, and $H_{0}=3 \Phi_{0} /\left(\pi^{2} \xi_{0} \lambda_{0}\right)$ is of the order the thermodynamic critical field of the system $\left(\Phi_{0}\right.$ is the flux quantum, $\xi_{0}$ the coherence length and $\lambda_{0}$ the penetration depth). Later, it was shown [3] that impurities alter this prediction, leading to an asymptotic $\delta \lambda \sim H^{2}$ dependence, but only at concentrations such that the linear term in the $H \rightarrow 0$ temperature dependence $\delta \lambda(T) \sim T$ is replaced by $\delta \lambda(T) \sim T^{2}$ 田 6 .

From an experimental standpoint, the search for confirmation of this fundamental manifestation of $d$-wave symmetry has been controversial. In 1995, Maeda et al. reported the first observation of a linear $H$ term on $\mathrm{Bi}_{2} \mathrm{Sr}_{2} \mathrm{CaCu}_{2} \mathrm{O}_{8}(\mathrm{BSCCO}-2212)$ films [7], but the results were questionable since they were obtained at relatively high temperatures, where the Yip-Sauls theory predicts a $\delta \lambda \sim H^{2}$. The clean $d$-wave nonlinear Meissner effect should be obtained, according to the simple theory, only for temperatures below a crossover $T \ll E_{\text {nonlin }}$, with $E_{\text {nonlin }}=v_{s} k_{F} \simeq\left(H / H_{0}\right) \Delta_{0}$ and $\Delta_{0}$ the gap maximum, giving typically $E_{\text {nonlin }} \simeq 1 K$ for the cuprates, for fields close to $H_{c 1}$, the lower critical field. Measurement of a linear- $H$ term was also claimed at much higher temperatures for $\mathrm{YBa}_{2} \mathrm{Cu}_{3} \mathrm{O}_{7-\delta}(\mathrm{YBCO})$ samples [8]. It is important to recall that several extrinsic effects can give rise to spurious field dependences, most importantly contributions from trapped vortices which enter near sample edges. An extrinsic contribution $\delta \lambda \sim \sqrt{H}$ to the effective penetration depth can arise from coupling of the $\mathrm{rf}$ field used in resonant coil experiments to the pinned vortices [9]. Such dependences were also found in measurements at much lower temperatures and on higher quality single crystals of $Y B C O$ by Carrington and Giannetta [10]. Finally, a measurement to detect higher-harmonic nonlinear modes in the ac response predicted on the basis of Yip-Sauls theory by Žutić and Valls [11, apparently less sensitive to the effects of trapped flux, failed to observe the predicted signal [12].

We therefore take the point of view that the available experimental data do not support the existence of a nonlinear Meissner effect in the best cuprate samples, and ask why. Our answer is that the effects of nonlocal electrodynamics, neglected in the work of Yip and Sauls, serve to cut off the (local) nonlinear Meissner effect as the field is lowered, replacing the linear- $H$ response with a quadratic one below a crossover field $H^{*}$. In most experimental geometries, this crossover is of the order of the lower critical field $H_{c 1}$, meaning the $\delta \lambda(H) \sim H$ behavior cannot generically be observed in the Meissner state. However, we show that nonlocal effects are negligible in geometries with currents parallel to a nodal direction.

Nonlocal electrodynamics. The Yip-Sauls description of the nonlinear Meissner effect is performed in the local limit, i.e. the $d$-wave Cooper pairs, although extended objects, are assumed to respond to the electromagnetic field at a single point at the center of the pair. Such an approximation is normally justified in classic type-II superconductors, where $\lambda_{0}$ is much larger than $\xi_{0}$. Although the cuprates are strongly type-II superconductors, with small in-plane $\xi_{0} \simeq \mathcal{O}(15 A)$ and large 
in-plane $\lambda_{0} \simeq \mathcal{O}(1500 A)$, effects of nonlocal electrodynamics are to be seen in several special situations, as a result of the gap nodes, along which the "effective coherence length" $v_{F} / \pi \Delta_{k}$ diverges [13]. Such effects were shown to significantly suppress the shielding supercurrents in measurements of the ac Meissner effect [15] in unconventional superconductors with line nodes, with application to heavy fermion materials. In the context of the cuprates, it was shown by Zucarro et al. [16] that significant variations in the electromagnetic response of clean $d$-wave superconductors were to be expected when nonlocal effects were included. But it was Kosztin and Leggett [13] who first pointed out that the very signature of a superconductor with line nodes, the linear- $T$ term in the dc penetration depth, was modified at the lowest temperatures 14. The crossover temperature below which this occurs is $E_{\text {nonloc }} \simeq \kappa^{-1} \Delta_{0}$, where $\kappa \equiv \lambda_{0} / \xi_{0}$ is the Ginzburg-Landau parameter. The characteristic crossover field defined by $E_{\text {nonloc }}=E_{\text {nonlin }}$ is given by $H^{*} \simeq \kappa^{-1} H_{0} \simeq H_{c 1}$. One might therefore expect that nonlocal effects "cut off" the nonlinear Meissner effect. This observation needs, however, to be supported by a full calculation, which we now sketch.

General current response. The current response for a $d$-wave superconductor may be derived from a full calculation of the BCS free energy for fixed external field $\mathbf{H}$ corresponding to vector potential $\mathbf{A}$. We have performed such a calculation (including both nonlocal and nonlinear effects analytically) under the assumptions of i) negligible spatial variations of the amplitude of the order parameter, $\Delta_{\mathbf{k}}(\mathbf{r})$; and ii) slow spatial variations of the superfluid velocity $\mathbf{v}_{s}$. The details of the derivation will be given elsewhere [17], but the final result for the Fourier component of the current in an infinite system at constant $\mathbf{v}_{s}$ is $\mathbf{j}(\mathbf{q})=-\mathcal{K}\left(\mathbf{q}, \mathbf{v}_{s}, T\right) \mathbf{A}(\mathbf{q})$, where

$$
\frac{\mathcal{K}\left(\mathbf{q}, \mathbf{v}_{s}, T\right)}{c /\left(4 \pi \lambda_{0}^{2}\right)}=1+\frac{2 T}{n m} \sum_{\omega_{n}, \mathbf{k}} \mathbf{k}_{\|}^{2} \frac{z_{\mathbf{k}}^{2}+\xi_{+} \xi_{-}+\Delta_{+} \Delta_{-}}{\left[z_{\mathbf{k}}^{2}-E_{+}^{2}\right]\left[z_{\mathbf{k}}^{2}-E_{-}^{2}\right]},
$$

with $\lambda_{0}=\sqrt{m c^{2} / 4 \pi n e^{2}}$ the local penetration depth at $T=0$ in linear response theory, $n$ the electron density, $m$ the electron mass, $c$ the speed of light, $\mathbf{k}_{\|}$the projection of $\mathbf{k}$ onto the surface, $z_{\mathbf{k}} \equiv i \omega_{n}+\mathbf{v}_{s} \cdot \mathbf{k}_{F}$, $E_{ \pm}^{2} \equiv \xi_{ \pm}^{2}+\Delta_{ \pm}^{2}, \xi_{ \pm} \equiv \xi_{\mathbf{k} \pm \mathbf{q} / 2}$, and $\xi_{\mathbf{k}}=\frac{k^{2}}{2 m}-\mu(\mu$ the chemical potential). This is the standard linear response result with all quasiparticle Matsubara energies $\omega_{n}$ modified by the semiclassical Doppler shift $\mathbf{v}_{s} \cdot \mathbf{k}_{F}$ [18]. For the real physical system in the Meissner state, a surface boundary is present and $v_{s}$ decays with the distance from the surface $y$. In the linear, local approximation, $v_{s}(y)=\left(e \lambda_{0} H / m c\right) \exp \left(-y / \lambda_{0}\right)$ decays exponentially and $v_{s}(y=0)$ is proportional to the external field, neither of which hold generally. But we may imagine the system to be subdivided into many layers, in each of which $v_{s}$ is roughly constant. Furthermore, we assume specular scattering surface boundary condition on the quasiparticle wavefunctions. In this case, the response is given by Eq. (2). The geometry which we study first is such that the magnetic field is along the $c$ axis and thus $\mathbf{v}_{s}$ lies in the $a b$ plane; the normal of the boundary plane $\hat{q}$ is also in the $a b$ plane, forming an angle $\theta$ with the $b$ axis. We will discuss the situation where $H$ is perpendicular to the $c$ axis later.

To a first approximation, we neglect the space dependence of $v_{s}$ and replace it by its value at the surface $v_{s} \simeq v_{s}(y=0)=e \lambda_{0} H / m c$ as given by the solution to the linear, local electrodynamics problem. Now we separate out the $T=0$ local, linear response as $\mathcal{K}\left(\mathbf{q}, \mathbf{v}_{s}, T\right)=c /\left(4 \pi \lambda_{0}^{2}\right)+\delta \mathcal{K}\left(\mathbf{q}, \mathbf{v}_{s}, T\right)$, and then define the nonlinear, nonlocal penetration depth to be

$$
\lambda_{\text {spec }}=\int_{0}^{\infty} \frac{H(y)}{H(0)} d y \simeq \lambda_{0}-\frac{8}{c} \int_{0}^{\infty} d q \frac{\delta \mathcal{K}\left(\mathbf{q}, \mathbf{v}_{s}, T\right)}{\left(\frac{1}{\lambda_{0}^{2}}+q^{2}\right)^{2}} .
$$

This expression has the virtue of reducing exactly to the nonlocal expression of Kosztin and Leggett [13] if the $\mathbf{v}_{s^{-}}$ dependence is neglected, and (qualitatively) to the nonlinear expression of Yip and Sauls [2] if the $q$-dependence is neglected. It is worthwhile reviewing the latter case. When $T \ll v_{s} k_{F}$ we obtain

$$
\frac{\delta \mathcal{K}\left(\mathbf{q} \rightarrow 0, \mathbf{v}_{s}, T\right)}{c /\left(4 \pi \lambda_{0}^{2}\right)}=-\frac{\zeta_{\theta}}{\sqrt{2}} \frac{v_{s} k_{F}}{\Delta_{0}}
$$

where $\zeta_{\theta}=\frac{1}{2} \sum_{l= \pm 1}|\cos \theta+l \sin \theta|^{3}$. Thus,

$$
\frac{\delta \lambda_{\text {spec }}^{(\text {loc })}}{\lambda_{0}}=\frac{\lambda_{\text {spec }}^{(\text {loc })}-\lambda_{0}}{\lambda_{0}} \simeq \frac{\zeta_{\theta}}{2 \sqrt{2}} \frac{v_{s} k_{F}}{\Delta_{0}} \simeq \frac{3}{2} \frac{\zeta_{\theta}}{\sqrt{2}} \frac{H}{H_{0}} .
$$

We recall that Yip and Sauls [2] and Xu et al. [3] defined penetration depth from its initial decay: $\lambda=$ $-H(0) /\left.[d H(y) / d y]\right|_{y=0}$ which is equivalent to the definition in Eq. (3) only in the linear limit. Using their definition we find that the prefactor $3 / 2$ in Eq. (5) is modified to 3 . At this stage, we may improve our approximation by restoring the spatially varying nature of $v_{s}$. We follow Yip et al. to solve directly a nonlinear London equation

$$
\nabla^{2} \mathbf{v}_{s}=\frac{4 \pi}{c} \mathcal{K}\left(0^{+}, \mathbf{v}_{s}, T\right) \mathbf{v}_{s}=\frac{\mathbf{v}_{s}}{\lambda_{0}^{2}}\left(1-\frac{\zeta_{\theta}}{\sqrt{2}} \frac{k_{F}}{\Delta_{0}} v_{s}\right),
$$

under the boundary condition $d v_{s} /\left.d y\right|_{y=0}=(e / m c) H$. Using the relation $d v_{s} / d y=(e / m c) H(y)$ and their definition for the penetration depth again, we see that $\delta \lambda / \lambda_{0}=2 \frac{\zeta_{\theta}}{\sqrt{2}} \frac{H}{H_{0}}$. So in the local limit we get qualitatively the same nonlinear correction to the penetration depth as Yip and Sauls [2], comparing to Eq. (11), but with a larger prefactor. This deviation arises from the perturbative treatment of the response of the superconductor to the $\mathbf{A}(\mathbf{q} \neq 0)$ modes in the present theory. The nonlinear effects obtained at $T \ll v_{s} k_{F}$, due to the Doppler shift coming from the coupling of the $\mathbf{A}(\mathbf{q}=0)$ mode to the quasiparticles, are nonperturbative results. A discussion of this point can be found in Ref. 17]. 
Low-energy scaling properties. Explicit analytical results for the full response in the approximation of constant $v_{s}$ may now be obtained by expanding $\delta \mathcal{K}$ for low energies, $v_{s} k_{F}, q v_{F}, T \ll \Delta_{0}$. A 2-parameter scaling relation may be derived,

$$
\begin{array}{r}
\delta \mathcal{K}\left(q, v_{s}, T\right)=-\frac{c}{8 \pi \lambda_{0}^{2}} \frac{T}{\Delta_{0}} \sum_{l= \pm 1} u_{\theta l}^{2} F_{\lambda}\left(\frac{\alpha w_{\theta l}}{T}, \frac{\varepsilon u_{\theta l}}{T}\right), \\
F_{\lambda}\left(z_{1}, z_{2}\right)=\frac{\pi}{4} z_{1}+\left[\ln \left(e^{z_{2}}+1\right)+\ln \left(e^{-z_{2}}+1\right)\right] \\
-\int_{0}^{z_{1}} d x\left[f\left(x-z_{2}\right)+f\left(x+z_{2}\right)\right]\left[1-\left(\frac{x}{z_{1}}\right)^{2}\right]^{1 / 2}
\end{array}
$$

with $\alpha=v_{F} q /(2 \sqrt{2}), \varepsilon=v_{s} k_{F} / \sqrt{2}, u_{\theta l}=|\cos \theta+l \sin \theta|$, $w_{\theta l}=|\sin \theta-l \cos \theta|$ and $f(x)=1 /\left(1+e^{x}\right)$. We consider primarily the limit $T \ll \alpha, \varepsilon$. It is straightforward to see that for $z_{2} \gg 1$

$$
F_{\lambda}\left(z_{1}, z_{2}\right) \simeq \begin{cases}z_{2} & z_{1} \ll z_{2} \\ \frac{\pi z_{1}}{4}+z_{2}\left(z_{2}^{2}+\pi^{2}\right) /\left(6 z_{1}^{2}\right) & z_{2} \ll z_{1}\end{cases}
$$

The scaling form (7) may now be inserted into (3) to find the penetration depth. Its field dependence turns out to be determined by the ratio $\alpha w_{\theta l} / \varepsilon u_{\theta l}$, with a typical $q$ set to $1 / \lambda_{0}$ in (3) by the denominator. If we define two new dimensionless parameters $h_{\theta l}=\left(u_{\theta l} / w_{\theta l}\right) h, l=$ \pm 1 , with $h \equiv m v_{s} \lambda_{0} \rightarrow(3 / \pi) \kappa H / H_{0}$, we get $\delta \lambda_{\text {spec }}=$ $\sum_{l= \pm 1} \delta \lambda_{\text {spec }}^{(l)}$, where

$$
\begin{gathered}
\frac{\delta \lambda_{\mathrm{spec}}^{(l)}}{\lambda_{0}} \simeq \frac{\pi}{4 \sqrt{2}} u_{\theta l}^{3} \kappa^{-1} h+c_{\theta l 1} \kappa^{-1} \frac{1}{h^{2}}+c_{\theta l 2} \kappa \frac{1}{h^{4}} \frac{T^{2}}{\Delta_{0}^{2}} \\
\text { for } h_{\theta l} \gg 1,
\end{gathered}
$$

with $c_{\theta l 1}=0.008 w_{\theta l}^{3}$ and $c_{\theta l 2}=0.006 w_{\theta l}^{3} / u_{\theta l}^{2}$, and

$$
\begin{array}{r}
\frac{\delta \lambda_{\mathrm{spec}}^{(l)}}{\lambda_{0}} \simeq \frac{\pi}{8 \sqrt{2}} d_{\theta l 1} \kappa^{-1}+d_{\theta l 2} \kappa^{-1} h^{2}+d_{\theta l 3} \kappa \frac{T^{2}}{\Delta_{0}^{2}} \\
\text { for } h_{\theta l} \ll 1,
\end{array}
$$

with $d_{\theta l 1}=0.5 u_{\theta l}^{2} w_{\theta l}, d_{\theta l 2}=1.1 u_{\theta l}^{4} / w_{\theta l}$ and $d_{\theta l 3}=$ $0.47 u_{\theta l}^{2} / w_{\theta l}$. The singularity which leads to the linear term in Eq. (9) if nonlocal effects are simply neglected is seen to be cut off by the constant term in Eq. (10). This happens at $h_{\theta l} \sim 1$. For $\theta$ not too close to a nodal value, $w_{\theta l}$ and $u_{\theta l}$ are of order unity for both $l= \pm 1$, and the crossover field may be simply determined from $h=1, H^{*} \sim \kappa^{-1} H_{0}$. The thermodynamical critical field $H_{c} \simeq H_{0}$ is the geometric mean $\sqrt{H_{c 1} H_{c 2}}$ of the upper critical field $H_{c 2}=\Phi_{0} /\left(2 \pi \xi_{0}^{2}\right)$ and the lower critical field $H_{c 1}=\Phi_{0} /\left(2 \pi \lambda_{0}^{2}\right)$. Thus the crossover field for such geometries is of the same order as the lower critical field, $H^{*} \simeq H_{c 1}$. Since the Meissner state is unstable to the Abrikosov vortex state for fields above $H_{c 1}$, the linear- $H$ term in $\delta \lambda$ is asymptotically unobservable in these geometries. In the Meissner state it is, in fact, replaced by a quadratic variation, as seen in (10). In Fig. 1, we have plotted the penetration depth calculated in Kosztin and
Leggett's geometry $\theta=0$, according to the exact scaling function given in (7), to explicitly exhibit this effect.

When $\theta$ is near any node, however, $u_{\theta l}$ can be much larger than $w_{\theta l}$ and the nonlocal energy scale much smaller than the nonlinear one, leading to a very small crossover field $H^{*} \simeq H_{c 1} w_{\theta l} / u_{\theta l} \ll H_{c 1}$. We conclude that the nonlinear Meissner effect may be observable in such special geometries, e.g. a sample with a dominant (110) surface and $H \| c$.

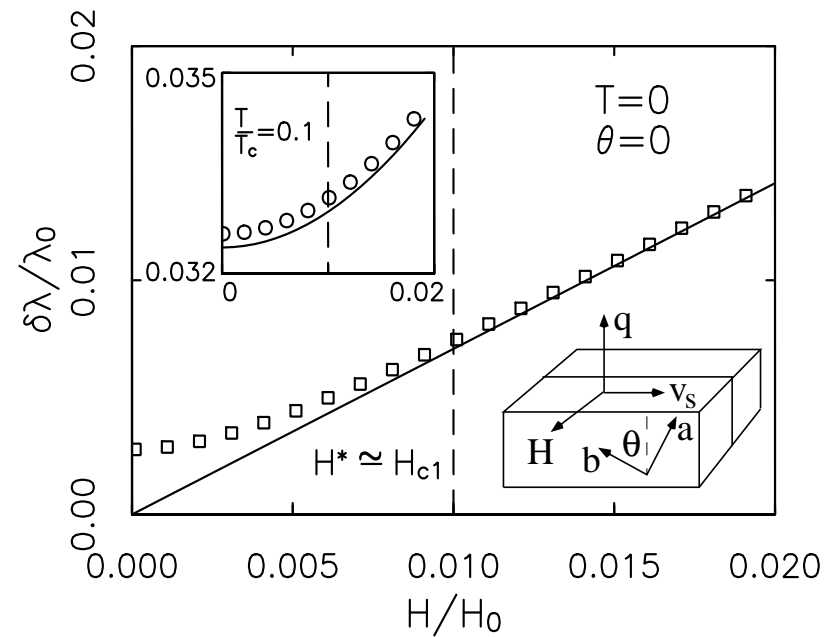

FIG. 1. Normalized penetration depth correction $\delta \lambda / \lambda_{0}$ vs. normalized magnetic field $H / H_{0}$ at $T=0$ for a sample with $\mathbf{H}$ parallel to a $(010)$ surface $(\theta=0)$ and Ginzburg-Landau parameter $\kappa=10^{2}$, hence lower critical field $H_{c 1} \simeq 10^{-2} H_{0}$. Squares: full response; solid line: nonlocal effects neglected. Insert: $T=0.1 T_{c}$. Circles: full response; solid line: nonlocal effects neglected. Dashed line: $H^{*} \simeq H_{c 1}$.

There is another special situation in the quasi-2D cuprates in which the characteristic energy of nonlocal effects becomes very small and the previous discussion might not be expected to apply. If we assume that the quasiparticles are strictly confined to the $a b$ plane and if the experimental configuration is dominated by a (001) surface with $\mathbf{H} \| a b$, then $E_{\text {nonloc }}^{(a b)}=\mathbf{q} \cdot \mathbf{v}_{F}=0$. In this case, however, the magnetic field is not screened $(\lambda \rightarrow \infty)$ 19. Whenever the quasiparticles are allowed to move along the $c$-direction we expect nonzero $E_{\text {nonloc }}^{(a b)}$ 20]. This can be demonstrated directly for any model of coherent transport along the $c$-axis. The materials of greatest current interest are the cuprate materials $Y B C O$ and $B S C C O-2212$. In the $Y B C O$ case, it is reasonable to treat the system as weakly-3D. The characteristic nonlocal energy is then $v_{F c} q \simeq \xi_{0 c} \Delta_{0} / \lambda_{0}$ much smaller than in the (010) case since the $c$-axis coherence length is $\xi_{0 c} \simeq 3 A$ as opposed to the in-plane coherence length of $\xi_{0} \simeq 15 A$. On the other hand, the lower critical field is also much smaller for this geometry, $H_{c 1}^{(a b)} \simeq \Phi_{0} /\left(2 \pi \lambda_{0} \lambda_{0 c}\right)$. Using $\lambda_{0 c} \simeq 0.5-0.8 \times 10^{4} A$, we again find a large crossover field $H^{*(a b)} \simeq\left(\xi_{0 c} / \xi_{0}\right) H^{*} \simeq$ $0.5-1 H_{c 1}^{(a b)}$, making it still impossible from a practical 
point of view to extract a linear- $H$ term [21].

The BSCCO system is so anisotropic that it is questionable whether the above argument applies. A full treatment of this problem awaits a generally accepted theory of the (incoherent) $\hat{c}$-axis transport in the normal state.

In conclusion, with the exception of the claim by Maeda et al. [7.8], all attempts to measure the nonlinear Meissner effect, a fundamental consequence of $d$-wave symmetry, have failed. As the order parameter symmetry is by now well-established in these materials, an explanation is required. To this end, we have considered the interplay of nonlinear and nonlocal effects in the electromagnetic response of a $d$-wave superconductor in the Meissner regime. This situation differs from the similar problem considered by Amin et al. [22 in the vortex phase due to the fact that the nonlinear and nonlocal energies $E_{n o n l i n}$ and $E_{n o n l o c}$ are not independent in their case, as a consequence of the fluxoid quantization condition. By contrast, in the Meissner state, while the nonlocal scale is set by intrinsic normal-state parameters, i.e. $T_{c}$ and $\kappa$, the nonlinear energy $E_{n o n l i n} \simeq v_{s} k_{F}$ is effectively tunable by an external field. We have shown that the nonlocal terms in the response generically cut off the nodal singularity in the nonlinear response, eliminating the linear- $H$ term in the penetration depth.

Neither the current theory nor the Yip-Sauls result, both based on a consideration of the shielding currents in the Meissner phase, can explain the results of Maeda et al. Maeda's results were obtained on samples which did not clearly exhibit a $\delta \lambda(T ; H \rightarrow 0) \sim T$ behavior in any range, and therefore should not show nonlinear effects even according to the Yip - Sauls theory. Furthermore, they were performed at temperatures of $9 \mathrm{~K}$ and above, and a linear- $H$ behavior was reported up to $50 \mathrm{~K}$. As we show in Fig. 1, even for temperatures as low as $0.1 T_{c}$, no linear term should be visible even if nonlocal effects are neglected. Therefore we must conclude that Maeda et al. observed an extrinsic effect, probably related to trapped vortices. Very recently, new high-resolution resonant coil measurements [10,23 on very clean $Y B C O$ samples observed (in some geometries) linear $H$ terms with magnitudes close to the Yip-Sauls prediction [10,23], but simultaneously measured qualitatively inconsistent temperature dependence. These authors also observed effects attributed to trapped vortices [10], and we therefore conclude that the linear term must be of extrinsic origin. Clearly, a test of the nonlinear effects which is independent of trapped flux is desirable. The harmonics of the nonlinear response predicted by Zuutić and Valls in principle provide such a test, but to date, a high resolution nonlinear transverse magnetization experiment designed to observe these resonances has failed to do so [12]. This null result is consistent with the current theory, but by itself cannot confirm it due to uncertainties regarding the identification of $H_{c 1}$ [12].

Finally, in all experiments up to now the orientation of the supercurrent was along the crystal axes. Our analysis suggests the best possibility to observe the effect is in geometries with $v_{s}$ along the nodal directions.

The authors are grateful to M. Franz, W. Hardy, and N. Schopohl for helpful communications, and particularly to R. Giannetta and A. Goldman for discussion of their unpublished results. Partial support was provided by NSF-DMR-96-00105 and the A. v. Humboldt Foundation.

[1] W. N. Hardy et al., Phys. Rev. Lett. 70, 3999 (1993).

[2] S. K. Yip and J. A. Sauls, Phys. Rev. Lett. 69, 2264 (1992);

[3] D. Xu, S. K. Yip and J. A. Sauls, Phys. Rev. B 51, 16233 (1995).

[4] F. Gross et al., Z. Phys. B 64, 175 (1986).

[5] M. Prohammer and J. P. Carbotte, Phys. Rev. B 43, 5370 (1991).

[6] P. J. Hirschfeld and N. Goldenfeld, Phys. Rev. B 48, 4219 (1993).

[7] A. Maeda et al., Phys. Rev. Lett. 74, 1202 (1995).

[8] A. Maeda et al., J. Phys. Soc. Jpn. 65, 3638 (1996).

[9] M. W. Coffey and J. R. Clem, Phys. Rev. Lett. 67, 386 (1991); E. H. Brandt, Phys. Rev. Lett. 67, 2219 (1991).

[10] A. Carrington and R. Giannetta, private communication.

[11] I. Zutić and O. T. Valls, cond/mat/9806288.

[12] A. Goldman, private communication.

[13] I. Kosztin and A. J. Leggett, Phys. Rev. Lett. 79, 135 (1997).

[14] Recently it was suggested that the rigorous linear $T$ dependence of the magnetic penetration depth would violate the third law of thermodynamics [N. Schopohl and O. V. Dolgov, Phys. Rev. Lett. 80, 4761 (1998)]. However, the nonlocal and nonlinear effects serve to cut off this linear $T$ dependence at low temperatures (Ref. 20]).

[15] W. O. Putikka, P. J. Hirschfeld, and P. Wölfle, Phys. Rev. B 41, 7285 (1990).

[16] C. Zuccaro, C. T. Rieck and K. Scharnberg, Physica C 235-240, 1807 (1994).

[17] M. -R. Li, P. J. Hirschfeld, and P. Wölfle, unpublished.

[18] K. Maki and T. Tsuneto, Prog. Theor. Phys. 27, 228 (1962).

[19] D. I. Glazman and A. E. Koshelev, Sov. Phys. JETP 70, 774(1990).

[20] P. J. Hirschfeld, M. -R. Li, and P. Wölfle, Phys. Rev. Lett. 81, 4024 (1998).

[21] For representative values of $\xi_{0}$ and $\lambda_{0}$, see S. L. Cooper and K. E. Gray, in Physical Properties of High Temperature Superconductors, IV, D. M. Ginsberg, ed., Singapore: World Scientific, 1994.

[22] M. H. S. Amin, I. Affleck, and M. Franz, Phys. Rev. B 58, 5848 (1998).

[23] C. P. Bidinosti et al., cond/mat/9808231. 